\title{
WIRELESS SENSOR NETWORKS CONGESTION AND ROLE OF ARTIFICIAL INTELLIGENCE
}

\author{
M.S. Doibale \\ Research Scholar, School of Computational Sciences \\ S.R.T.M.U. Nanded, Maharashtra, India \\ Dr. G. D. Kurundkar \\ Gurubuddhiswami Mahavidyalaya, Purna, \\ Dist. Parbhani, Maharashtra, India.
}

\begin{abstract}
The occurrence of the congestion has an extremely deleterious impact on the performance of Wireless Sensor Network (WSNs). Some novel blockage control method utilizing computerized reasoning for remote sensor systems. Remote Sensor Systems are a class of remote systems estimated for checking objective and ecological wonders. Our paper provides opening to wireless sensor network as well as for artificial intelligence, which aims to act both proactively, in order to avoid the creation of congestion in WSNs, and reactively, so as to mitigate the diffusion of upcoming congestion through alternative path routing. We make use of Artificial Intelligence (AI) which play an important role in our society, give rise to systems that can manage themselves. Many advanced AI techniques can be utilized to improve the performance and reliability. Investigating the AI algorithm applied to WSN may improve network management, security or routing in WSN which may result in a more reliable network. We will look at AI algorithm applied in WSN and discuss the possible use of these AI in WSN to address the WSN challenge and improve its performance and reliability. And due to the constraint on data processing and power consumptions, use of artificial intelligence has been historically discarded in these kind of network. The use of the AI in WSN makes system error free, fast and efficient in almost many aspects. This paper attempts to encourage the use of artificial intelligence techniques in wireless sensor nodes.
\end{abstract}

Keyword head: WSN (Wireless Sensor Network), control framework, booking, strength, streamlining, counterfeit consciousness, stream control, AI, security, routing, and traffic management.

Cite this Article: M.S. Doibale and Dr. G. D. Kurundkar, Wireless Sensor Networks Congestion and Role of Artificial Intelligence, International Journal of Computer Engineering and Technology, 10(2), 2019, pp. 60-66.

http://iaeme.com/Home/issue/IJCET?Volume=10\&Issue=2 


\section{INTRODUCTION}

Wireless sensor networks (WSNs) which are generally composed of one or more sinks, thousands of sensor nodes scattered in a physical space. With integration of data sensing, computation, and wireless communication, the sensor nodes can understand physical data, then operates the crude data, and furthermore report the required information to the sink. The common task of sensor node is to collect the information from the scene of event and send the data to a sink node. But the sensors are small, so they have less computation and calculated resources essential information to the sink. These nodes are able to understand, scale, and collect data from the surrounding and based on some local decision processes, they will transmit the sensed data to user. And this sensor will works on AI technics.

WSNs can be used in many applications such as habitat monitoring, security surveillance, target tracking, medical application and etc. Wireless sensor network (WSN) is a high degree of cross-disciplinary, highly integrated knowledge on network communication, and is a forefront study hot spot in the world.

In this work, the attributes, benefits and cons of clustering based on cluster count, diversity, cluster overlapping, and role of cluster head, objective of sensor node grouping and methodology with that of Artificial Intelligence algorithms is studied.

\section{CONGESTION AND USE OF AI}

In WSN, the issue of congestion adversely affects almost every Quality of Service (QOS) parameter. Congestion usually happen in the sensors-to-sink direction when packets are transported in a many-to-one manner [9]. Congestion is reasoned to occur when any node or link get the data more than its sustained capacity. To improve the network performances like throughput of system, to reduce the time delay through the protocols design, route algorithm choose, data integration and load balancing, and so on. [6]. Figure 1 shows how the operational performance of the network gets low in congestion mode. 90 percent of the traffic data is caused by the resource which utilizes the TCP protocol in transfer layer. Working of AI in WSN protocols is also studied and reported in the next section.

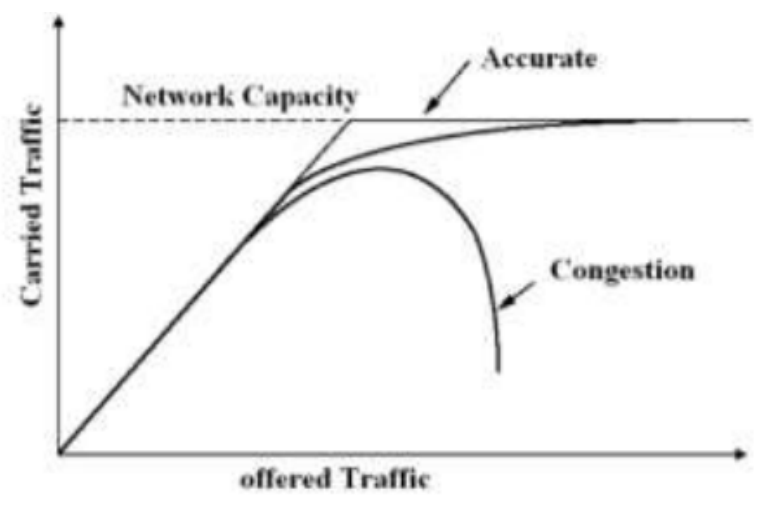

Figure: 1. Network Performance in Congestion mode.

\subsection{Congestion types in Wireless Sensor Networks}

\subsubsection{Node-level congestion:}

The node-level congestion is very normal in conventional networks. It is caused by a buffer overflow in the node and may result in packet loss, and also to gain in queuing delay [5]. 


\subsubsection{Link-level congestion:}

The link-level congestion normally occurs in a specific area, in network severe clashes could occur when multiple active sensor nodes which are within scope of one another efforts to transfer at the same time. Packets that leave the buffer might fail to reach the next hop as a consequences of a clashes. This kind of congestion leads to decrease both link utilization and overall throughput, while increasing both packet delay and energy waste [9].

\subsection{Congestion control schemes}

Congestion control schemes found in the literature can be separated into two main classes specifically:

(a) Centralized Congestion Control Schemes containing routing protocols aided with congestion control.

(b) Distributed Congestion Control Schemes contains buffer based and cross layer congestion control schemes [11].

Recently researchers have considered using Artificial Intelligence in WSN approach for power saving, improve routing and security in WSN. Many scientists use machine learning as a technique for Artificial Intelligence. AI or machine learning are important in WSN mainly because:

Dynamic environments transform over period and sensor nodes are used to supervise these changes, sensor nodes must modify and operate productively.

Data is sometimes gathered from sites that are unreachable, risky and unpredictable thus self-calibration network will be essential to study and govern the data and its flow. The functioning and use of AI techniques is illustrated in next section.

\section{CONGESTION CONTROL IN AI BASED IN ROUTING AND TRAFFIC MANAGEMENT}

Congestion controlling methods for traffic entry controlling at the nodes, so as to avoid WSN collapse due to overflow of any of the treatment or packets from any of the intermediate nodes and networks and by fetching resource shortening steps, which are like reducing the rate of transmitting packets. Two general ways to manage congestion are Traffic Rate control and Resource Management

\subsection{Traffic rate control}

In the traffic rate control method, congestion is monitored by reducing number of packets injected into wireless sensor networks. It is divided into (AIMD) Additive Increase Multiplicative Decrease or a rate based method. In AIMD it is to be verify that total network ready bandwidth will gradually increases size of the congestion window. Throughout congestion the protocol diminishes the congestion window considerably, which is to be monitored.

\subsection{Resource management}

The traffic control method is not suitable for event based application. To overcome this method, a substitute method called resource control. Here when the network congested data packets are send from alternative paths to the sink from paths which are not congested. With this method not only traffic control is avoided but also, as all data packets have a great opportunity to reach the sink. At the same time special care is taken in order to meet the performance requirement like packet travel time, avoidance of loops etc. [13] 


\section{WORKING OF AI}

Artificial Intelligence has become an interesting topic in almost all research areas taken by scientist and engineers. It is a topic for the intelligence of machines or machine learning. In this section, we discuss some of the application that researchers have used AI algorithms or techniques as an approach to WSN with an objective to utilize them in AI based WSN.

Below Figure 2 shows the working of AI. AI works on trained dataset environment which learns from this input data and proceeds, manipulate new input data with reference of this values. Means machine are skilled to response and understand from datasets. Here the dataset is divided into three sets mainly in $70 \%$ training, $15 \%$ validation, $15 \%$ testing parts. And results are very accurate as desired with 0.0004 Mean-Square error (MES).

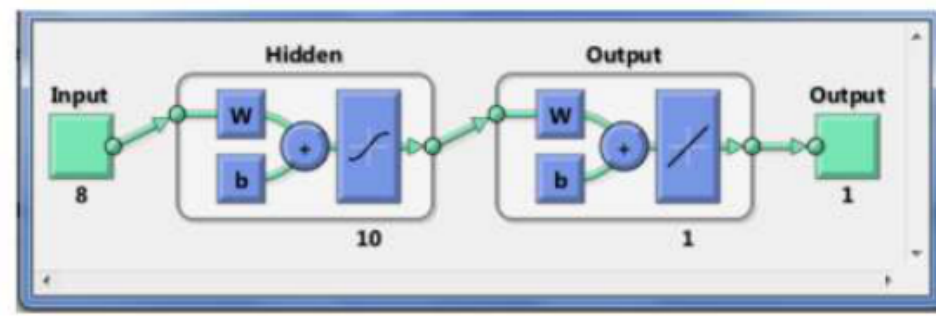

Figure: 2. Designed AI diagram for congestion control

Here some of the efficient algorithms based on AI are introduced to approach routing and QoS traffic classification in WSN. First one is Back propagation neural network (BPNN) which is used to achieve real time dynamic load balance and has decreased latency by $19.3 \%$ compared to other method like static round robin methods. Ant Colony Optimization (ACO) is another algorithm that is based on how ants behave to find the best route to get from their food supply to the nest. The SDN application runs ACO algorithms on a weighted graph in the SDN controller, where the weights are the loss rate and delay experienced in each network device. ACO compared to the shortest path algorithm has achieved $24.1 \%$ increase for the Quality of Experience $(\mathrm{QoE})$ value. After this, in next section the use of AI in communication and network training part, energy consumption methods, etc. are discussed one by one.

\subsection{WSN Communication With Reference To AI}

Artificial Intelligence system within WSN is also known as an intelligent wireless sensor. It modifies its internal behavior to optimize its ability to collect data from the physical world and communicate it in a responsive manner, to a base station or to a host system. The functionality of intelligent sensor includes: self-calibration, self-validation, and compensation. The selfcalibration means that the sensor can monitor the measuring condition to decide whether a new calibration is needed or not. Self-validation applies mathematical modelling error propagation and error isolation or knowledge-based techniques. The self-compensation makes use of compensation methods to achieve a high accuracy. The types of artificial intelligence techniques widely used in communication are: KT403 WIFI Communications system, KT314 3G Wireless Communications and Tracking System and KJ272 ZigBee Communication system. The use of these Artificial intelligence techniques plays a key role in building intelligent sensor structures for communication.

\subsection{Network Training For TCP Control Using AI}

One of the method to control the TCP is discussed here. The linear artificial network is studied, in this one can not only produce binary output, but also generate analog output of congestion. Here the output of linear transfer function can be of any value. Linear AI network diagram as shown in Figure 3 and $x$ is the input, $w$ is weight, $y$ is the linear output, and $q$ is a binary output. 
Subsequent use of RTT (Round Trip Time) and the current throughput of TCP connections as the main inputs of the linear AI network, and based on the results train the network to get the decision boundary which is used to predict the current congestion state [2].

We use LMS (Least Mean Square) learning algorithm for the linear AI network. We also name it as Delta rule. The procedure is as follows:

Step 1: Defining variables and parameters;

Step 2: Initialization. Giving a smaller initial random value to the weight;

Step 3: Inputting the samples and calculating the difference of the wanted output and the real output;
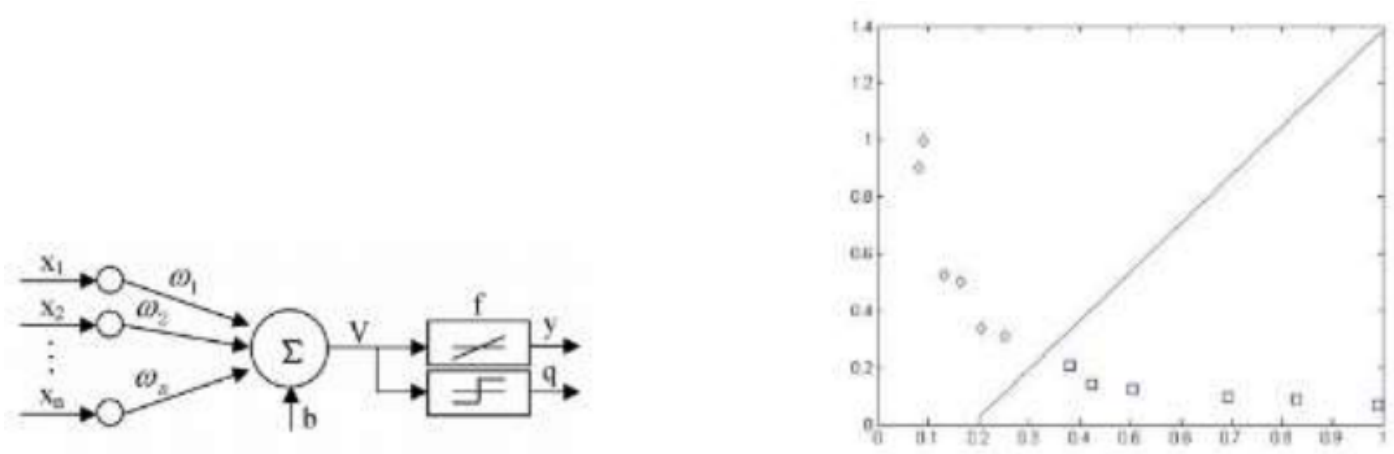

Figure: 3. Linear Artificial network Figure: 4. Decision boundary for predicting congestion

$$
W(n+1)=w(n)+\eta X T(n) d(n)
$$

Step 4: Adjusting the weights. Continuing to calculate Eq. 1 in accordance to the error calculated in the above step. In the Eq. $1, w(n)$ is weight, $\eta$ is the learning rate, $X T(n)$ is the sample inputted, and $d(n)$ is the difference between the expected value and the actual value:

Step 5: Ending if it's converged.

$$
y=\frac{1}{2} * w(2)-\frac{w(1)}{w(2)} * x-\frac{w(3)}{w(2)}
$$

According to the steps above, the maximum learning rate $\eta_{\text {is calculated firstly. Then } 12}$ samples are inputted. The desired output values are set as six continuous 0 and six continuous 1. Number 0 indicates non-congestion and 1 indicates congestion. Then we start to teach the linear neural network. At last the training result $w(n)$ is substituted into the Eq. 2. 1/2 in the Eq. 2 is a threshold of congestion. Eq. 2 is the decision boundary we get for predicting congestion, and the result as shown in Figure 4. If the coordinate of the RTT and the throughput of the current TCP connection is above the decision boundary, it indicates that the present network state is good and congestion does not occur. On the contrary, if the coordinate is below the decision boundary, it shows that congestion is happening or will happen. If so, the CWND value will not increase along with the received acknowledgements, but keep constant to avoid the congestion by reducing the data transfer rate. If the network status changes better, the coordinate returns to the above, then congestion notice is relieved, and continues to perform the original TCP congestion control algorithm [2] 


\subsection{Energy consumption Topology}

Routing is very important form energy point, as the total work flow as well as energy consumption is calculated based on this routing techniques. In general, routing in WSNs can be divided into at-based routing, hierarchical-base routing, and location-based routing. In this paper we study networks where all nodes are supposed to be assigned equal roles or functionalities. In this sense, at-based routing is best suited for this kind of networks. Among all the existing at routing protocols, we have chosen directed diffusion and Energy-Aware Routing (EAR) to evaluate the influence of the use of AI techniques.

EAR is similar to directed diffusion. In directed diffusion, sensors measure events and create gradients of information in their respective neighborhoods. The base station request data by broadcasting interests. Each sensor that receives the interest sets up a gradient toward the sensor nodes from which it has received the interest. This process continues until gradients are set up from the sources back to the base station. Nevertheless it differs in the sense that it maintains a set of paths instead of maintaining or enforcing one optimal path at higher rates. These paths are maintained and chosen by means of a certain probability. The value of this probability depends on how low the energy consumption that each path can achieve is. By having paths chosen at different times, the energy of any single path will not deplete quickly. Hence these are effective manner of energy consumption.

\section{CONCLUSION}

The paper discourses Artificial Intelligence based techniques for Novel Conjunction Control in wireless sensor networks. Congestion is occurred due to node or link get the data more than its capacity. The WSN embedded with Artificial Intelligence methods help in system not only in terms of system performance enhancement but also help in resolving network issues.

The two general ways to control congestions are by controlling the traffic rate and by managing the resource. We study that, the routing topology effect on the energy consumption of a sensor node. The parameters like energy optimization, cluster formation technique, inter network communication improvement, sensor development and there performance improvement, along with overall lifespan enhancement of WSNs are considered for study and improved after training WSN with AI. The work of this paper mainly focused towards designing energy efficient congestion control protocol which includes one or more features like decentralized, self-adapted, distributed, scalable, autonomous, generalized and secured congestion control strategies.

\section{REFERENCES}

[1] Andrés Felipe Luque Calderón, et al, Predicting Traffic through Artificial Neural Networks, Contemporary Engineering Sciences, Vol. 10, issue no. 24, pages1 195 - 1209, 2017.

[2] Lei Niu, et al, Applying the Linear Neural Network to TCP Congestion Control, 5th International Conference on Advanced Design and Manufacturing Engineering (ICADME), pages 558-562, 2015.

[3] Najme Tanzade Panah Et Al, A New Predictive Model for Congestion Control In Wireless Sensor Networks, Journal of Engineering Science and Technology, Vol. 12, pages 1601 1616, 2017.

[4] Wan, C, et al, Energy-efficient congestion detection and avoidance in sensor networks. ACM Transactions on Sensor Networks, vol. 7, pages 32.1-32.31, 2011.

[5] Monica R. Mundada, et al, A Survey of Congestion in Wireless Sensor Networks, International Conference on Advances in Human Machine Interaction (HMI - 2016), 2016. 
[6] Qixiang Pang, et al, Reliable data transport and congestion control in wireless sensor networks, Int. J. Sensor Networks, Vol. 3, No. 1, 2008.

[7] Jaspreet Kaur, et al, A Review on Deployment schemes in Wireless Sensor Network, Advances in Computational Sciences and Technology ISSN:0973-6107 Volume 10, pages 1161-1172, 2017.

[8] Marcus Okunlola Johnson et al, A Wormhole Attack Detection and Prevention Technique in Wireless Sensor Networks, International Journal of Computer Applications, Vol. 174, pages 1-8, 2017.

[9] Raheleh Hashemzehi et al, Congestion in Wireless Sensor Networks and Mechanisms for Controlling Congestion, Indian Journal of Computer Science and Engineering (IJCSE) ISSN: 9076-5166 Vol.4 No.3 2013.

[10] Heikalabad S. R, et al, DPCC: Dynamic Predictive Congestion Control in Wireless Sensor Network, International Journal of Computer Science (IJCSI), Vol. 8(1), pages 1694-0814, 2011.

[11] Yongmao Ren, et al, A Novel Congestion Control Algorithm for High Performance Bulk Data Transfer, IEEE International Symposium on Network Computing and Applications, 2009.

[12] Syed Afsar Shah, et al, Congestion control algorithms in wireless sensor networks: Trends and opportunities, Journal of King Saud University - Computer and Information Sciences, pages 236-245, 2017.

[13] K. Sumathi, et al, A Survey on Congestion Control in Wireless Sensor Networks, International Journal of Computer Applications Volume 147 - No.6, 2016.

[14] Dr.Rekha Purohit, et al, Role of Wireless Sensor Networks in Communication with Artificial Intelligence System, International Journal of Wireless and Mobile Communication for Industrial Systems Vol. 3, No. 2, pages 33-38, 2016. 